\title{
Strategies for Managing Chronic Pain: Case of a Skilled Orthopaedic Physician and Mini-Review
}

\author{
Tatsunori Ikemoto1,2, Young-Chang P. Arai1,2, Makoto Nishihara1, Takahiro Ushida1,2 \\ ${ }^{1}$ Multidisciplinary Pain Center, Aichi Medical University, Nagakute, Aichi, Japan \\ ${ }^{2}$ Institute of Physical Fitness, Sports Medicine and Rehabilitation, School of Medicine, Aichi Medical University, \\ Nagakute, Aichi, Japan \\ Email: tatsunon31-ik@umin.ac.jp
}

Received 9 April 2015; accepted 3 May 2015; published 6 May 2015

Copyright (C) 2015 by authors and Scientific Research Publishing Inc. This work is licensed under the Creative Commons Attribution International License (CC BY). http://creativecommons.org/licenses/by/4.0/

(c) (i) Open Access

\section{Abstract}

Epidemiological surveys have recently revealed a high prevalence of chronic musculoskeletal pain in Japan; however, $\mathbf{3 0 \%}$ of the patients in the survey were not satisfied with their pain treatment. This indicates that standard strategies in the management of chronic pain are poorly shared among physicians in Japan. Herein we report a case of a patient with intractable chronic pain who is a skilled orthopaedic physician. A 43-year-old man who was a skilled orthopaedic surgeon presented at our center complaining of severe buttock pain especially around the right hip region for more than three years. At begging of pain onset, he was diagnosed with femoacetabular impingement syndrome (FAI) with labral tear. Despite biophysical interventions including twice surgeries and alternative conservative treatment, his pain persisted, and he occasionally had to take a day off work due to the severe pain. Therefore we had to evaluate his pathological condition using a multidimensional approach based on a biopsychosocial model. We had provided him with cognitive behavioral therapy (CBT) approach, and simultaneously suggestion for short leaving from work. Three months after the start of CBT training, his disabilities had begun to improve. About six months later, he could continue to do his work. Finally, 19 months have passed since we started implementing the CBT approach; he has regained both his previous work-life balance and his health, although the pain has not completely subsided. In conclusion, we think it is important for physicians treating chronic pain to learn the management strategies for chronic pain and to reconsider their management policy when conventional biomedical interventions were not succeeded, even in cases where medication and surgical intervention are warranted.

\section{Keywords}

Chronic Pain, Cognitive Behavioral Therapy, Biomedical, Biopsychosocial 


\section{Introduction}

Epidemiological surveys have recently revealed a high prevalence of chronic musculoskeletal pain in Japan [1]-[3]. Therefore, there are a large number of orthopedic physicians treating patients with chronic pain. However, $30 \%$ of the patients in the survey were not satisfied with their pain treatment [1], and as a result they changed medical institutions to seek better treatment. In fact, most patients visiting our pain center had already been treated at several orthopaedic clinics. This indicates that standard strategies in the management of chronic pain are poorly shared among orthopaedic physicians. Hence we questioned whether orthopaedic physicians suffering from chronic pain as a patient would be able to treat themselves with appropriate interventions.

Herein we report a case of a patient with intractable chronic pain who is a skilled orthopaedic physician, of course with a license approved by Japanese orthopaedic association.

\section{Case Report}

A 43-year-old man who was a skilled orthopaedic surgeon and who also had several scientific articles published in MEDLINE (http://www.ncbi.nlm.nih.gov/pubmed) presented at our pain center complaining of severe buttock pain especially around the right hip region.

Three years and four months prior to visiting our pain center, he felt right hip pain while twisting his body when playing golf. After this injury, he was diagnosed with femoacetabular impingement (FAI) syndrome with labral tear without bone insult by using enhanced magnetic resonance imaging (MRI) (Figure 1). Despite the conservative treatment that he underwent such as muscle strengthening exercises and medications, the right hip pain persisted and had still not subsided two year after the onset of injury. After he had adequately discussed the pathology with a physician in charge and his colleagues, he decided to undergo arthroscopic hip surgery for mixed FAI lesions [4] [5]. On the surgery, operator found the labral tear in the part of bridging to capsule and the lesion was repaired.

After this surgery, however, the hip pain did not improve, and furthermore he experienced numbness in the right leg along the sciatic nerve and left hip pain without significant lesions in lumbar MRI which could explain these symptoms (Figure 2). Of course, possible disorders including spinal lesion such as L5/S lateral herniation,

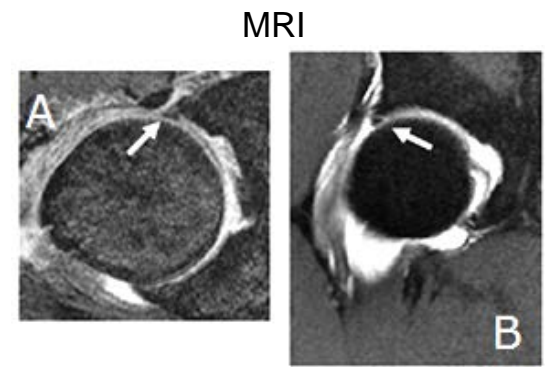

Figure 1. Magnetic resonance image of right hip joint. Both oblique planes (A, B) revealed a labral tear (arrow head) of the right hip joint.

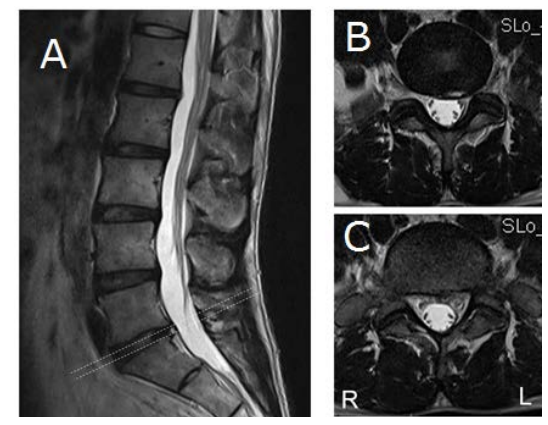

Figure 2. Magnetic resonance image of lumbar spine. Both sagittal (A) and axial at $5 /$ Slevel $(B, C)$ planes did not show narrow canal or intervertebral disc prolase, although there was mild degeneration. 
sciatic neuritis and piriformis syndrome were excluded by specialists in both spine surgery and neurology. He continued the rehabilitation exercises around the trunk and hip muscles, while simultaneously using several analgesic agents; not only NSAIDs but also antidepressants, anticonvulsants, and opioids which are widely used for chronic pain [6]. Nevertheless, his condition deteriorated and he occasionally had to take a day off work due to the severe pain.

Eight months after the primary surgery, he underwent a second arthroscopic hip surgery. At that time, the operator discovered a recurrence of the labral tear, hence the lesion was repaired again. After the second surgery, unfortunately his condition remained unchanged and he was therefore classified as having intractable chronic pain. Eight months later, he visited our pain center (Table 1).

On the first visit to the pain center, we had to evaluate his pathological condition using a multidimensional approach based on a biopsychosocial model [7] because he had disabilities for not only work but also daily living although he could walk without requiring any assistance. Physical examination and some images revealed no significant evidence of organic tissue injury as pointed previously, compatible with intractable chronic pain syndrome without apparent organic damage.

We evaluated the patient using an assessment battery of standardized self-report measures, demographics, symptoms, history and duration. The following instruments were used to assess pain: numerical rating scale (NRS) for pain severity, the Pain Disability Assessment Scale (PDAS) for the degree of the impact of painrelated disabilities on lifestyle [8] [9], the Hospital Anxiety and Depression Scale for assessing anxiety and depression (HADS Anxiety and HADS Depression) [10] [11], the Pain Catastrophizing Scale (PCS) for measuring catastrophizing due to pain [12] [13] and the EuroQol 5 Dimension (EQ-5D) for assessing QOL [14] [15]. During the first consultation, I told the patient that many things were impeding him from doing his work and they must have had an influence on his pain without apparent organic damage, and we provided some information about analgesic agents. For three months, no other interventions were taken for this patient. Subsequently, his condition neither improved nor showed signs of exacerbation (Table 2). We discussed his biopsychosocial con-

Table 1. Patient's history prior to visiting our pain center.

\begin{tabular}{cl}
\hline Time course & \\
\hline Onset & Rt hip injury due to sprain. Diagnosed as femoacetabular impingement syndrome with labral tear \\
24 months & Arthroscopic arthroplasty of hip joint, repair of labral tear \\
27 months & Absent from work due to chronic pain \\
32 months & Revision of arthroscopic arthroplasty of hip joint, repair of labral tear \\
\hline
\end{tabular}

Table 2. Assessment battery.

\begin{tabular}{|c|c|c|c|}
\hline \multirow{2}{*}{ Category } & \multicolumn{3}{|c|}{ Time course } \\
\hline & First visit & 3 months & Final (27 months) \\
\hline \multicolumn{4}{|l|}{$N R S$} \\
\hline Min & 3 & 1 & 0 \\
\hline $\operatorname{Max}$ & 8 & 8 & 2 \\
\hline Average & 5 & 6 & 1 \\
\hline PDAS & 18 & 16 & 0 \\
\hline \multicolumn{4}{|l|}{$H A D S$} \\
\hline Anxiety & 10 & 8 & 2 \\
\hline Depression & 10 & 9 & 2 \\
\hline \multicolumn{4}{|l|}{ PCS } \\
\hline Rimination & 5 & 10 & 2 \\
\hline Magnification & 4 & 3 & 0 \\
\hline Helplessness & 7 & 7 & 1 \\
\hline Total & 16 & 20 & 3 \\
\hline EQ-5D & 0.661 & 0.535 & 0.768 \\
\hline
\end{tabular}

Increase in scores of NRS, PDAS, HADS and PCS indicates more distress for patient, on the contrary increase in score of EQ-5D indicates improvement in quality of life in patient. The time course showed that condition in patient did not change at three months after first visit, whereas after start of cognitive behavioral approach, his distress was gradually alleviated as observed in final condition. Abbreviations; NRS: numerical rating scale, PDAS: Pain Disability Assessment Scale, HADS: Hospital Anxiety and Depression Scale, PCS: Pain Catastrophizing Scale, EQ-5D: EuroQol 5 Dimension. 
dition at team conferences attended by three orthopaedic physicians, an anesthesiologist, a psychologist, a nurse and some physical therapists. Our team conference suggested the need for a combination of an environmental change of work and a cognitive behavioral therapy (CBT) approach due to the fact that the patient had many duties and responsibilities at work, in addition to weekly night duty. Then, I informed him about our management policy.

He decided to take a leave from work eight-month after the first visit. I then starting using the CBT approach with this patient and through the change in the aim of treatment, he started to confront pain behavior even with the discomfort. For example, since he felt much distress sitting on a chair even for several minutes, he began to train himself to sit on a chair for a longer period of time and recorded it in his diary. His status was always confirmed by e-mail, but sometimes we discussed it face to face.

Three months after the start of CBT training, his sitting time had gradually increased although his buttock pain had not improved. Moreover, from that time, he started jogging once or twice a week at a level which did not exacerbate his hip pain, and then he was able to return to work without night duty on six month after leave from work. A few months later, he could continue to do his work without absence and was able to be on night duty.

Currently, 27 months have now passed since the first visit or 19 months since we started implementing the CBT approach. His condition finally improved, including disabilities of ADL (PDAS), mood (HADS), pain catastrophizing (PCS), QOL (EQ-5D), and pain intensity (Table 2). Now, he has regained both his previous work-life balance and his health, although the pain has not completely subsided.

\section{Discussion}

In the present report, we would like to emphasize two important issues; first of all, some orthopaedic physicians still appear to lack a biopsychosocial concept of chronic pain and secondly it is important to have a strategy to manage chronic pain.

In this case, the patient was a skilled orthopaedic physician with a license approved by the Japanese orthopaedic association and someone possessing scientific knowledge with several international publications in MEDLINE. Moreover, most of his fellow physicians and colleagues worked in scientific institutions such as university hospitals. Nevertheless, none of these physicians could alleviate his condition of chronic pain and finally gave up treatment. This reflects a poor level of knowledge about the concept for chronic pain among orthopaedic physicians, even in university hospitals.

In Japan, most of orthopaedic physician learn how to look for the cause of pain or how to eliminate pain in an educational program for getting a license approved by the Japanese orthopaedic association. Most of educational programs are based on biomedical, organ-based perspective focuses on disease mechanism. Hence, these educational backgrounds may lead them to be lack of knowledge for strategy to manage chronic pain. In addition, it is difficult for orthopaedic physician to run such CBT approach profitably under Japan's health insurance system because the insurance is only available for the intervention based on biomedical thesis. As a result, 30\% of chronic pain patients in the survey were not satisfied with their treatment [1].

Therefore, since 2011, a research group of the Ministry of Health, Labor and Welfare in Japan has begun to make educational materials regarding chronic pain for physicians, dentists and physical therapists respectively, although the materials were only completed one year ago.

We think that surgeries for labral tear in this case were pertinent because he certainly experienced organic damage in hip joint observed in MRI and surgical findings, however, treatment strategy after first surgery had to be considered as a biopsyosocial model [16].

Chronic pain, generally defined as pain persisting for more than 3 - 6 months, entails various problems that affect every aspect of quality of life, sleep, social functioning, and activities of daily living [17]. Patients with chronic pain fall into a vicious circle in which these psychological and social factors complicate their condition. In such cases, therapeutic approaches based on CBT using a multidisciplinary approach are recommended [16] [18]. CBT seeks to deepen the patient's understanding of his/her own pain and teach self-control and coping strategies in order to encourage behavioral modifications that allow the patient to better confront his or her pain and improve QOL as well [17]. We previously showed the efficacy of the CBT approach on patients with chronic pain using a group program [19].

In this case, although it took more than six months, we succeeded in helping a patient escape from a vicious circle of chronic pain by using both the CBT approach and through reminding him of self-control and coping 
strategies. These strategies are thought to be essential in order to confront chronic pain; however we think it may be still hard for both the physicians and patients to agree on these strategies in Japan because National Health Insurance is designed for people to have access to treatments such as medication, surgery, radiation and manipulation.

Hence, we think it is important for physicians treating chronic pain to learn the management strategies for chronic pain and to reconsider their management policy when conventional biomedical interventions were not succeeded [20], even in cases where medication and surgical intervention is warranted.

\section{Conclusion}

We treated a skilled orthopaedic physician with intractable chronic pain who has a license approved by the Japanese orthopaedic association. After the start of CBT training, his disabilities had begun to improve, and finally he has regained both his previous work-life balance and his health, although the pain has not completely subsided. We think that two surgeries for labral tear in this case were pertinent because of organic insult in hip joint observed in MRI and surgical findings; however, treatment strategy after first surgery had to be considered as a biopsychosocial model. Hence, we think it is important for physicians treating chronic pain to learn the management strategies for chronic pain and to reconsider their management policy when conventional biophysical interventions were not succeeded, even in cases where medication and surgical intervention are warranted.

\section{Acknowledgements}

We wish to thank the patient for providing us with both the details regarding his own patient history as well as several images concerning the disease.

\section{References}

[1] Nakamura, M., Nishiwaki, Y., Ushida, T. and Toyama, Y. (2011) Prevalence and Characteristics of Chronic Musculoskeletal Pain in Japan. Journal of Orthopaedic Science, 16, 424-432. http://dx.doi.org/10.1007/s00776-011-0102-y

[2] Nakamura, M., Nishiwaki, Y., Ushida, T. and Toyama, Y. (2014) Prevalence and Characteristics of Chronic Musculoskeletal Pain in Japan: A Second Survey of People with or without Chronic Pain. Journal of Orthopaedic Science, 19, 339-350. http://dx.doi.org/10.1007/s00776-013-0525-8

[3] Nakamura, M., Nishiwaki, Y., Sumitani, M., Ushida, T., Yamashita, T., Konno, S., Taguchi, T. and Toyama, Y. (2014) Investigation of Chronic Musculoskeletal Pain (Third Report): With Special Reference to the Importance of Neuropathic Pain and Psychogenic Pain. Journal of Orthopaedic Science, 19, 667-675. http://dx.doi.org/10.1007/s00776-014-0567-6

[4] Ito, K., Minka 2nd, M.A., Leunig, M., Werlen, S. and Ganz, R. (2001) Femoroacetabular Impingement and the CamEffect. A MRI-Based Quantitative Anatomical Study of the Femoral Head-Neck Offset. Journal of Bone \& Joint Surgery British Volume, 83, 171-176. http://dx.doi.org/10.1302/0301-620X.83B2.11092

[5] Bedi, A. and Kelly, B.T. (2013) Femoroacetabular Impingement. Journal of Bone \& Joint Surgery American Volume, 95, 82-92. http://dx.doi.org/10.2106/JBJS.K.01219

[6] Finnerup, N.B., Otto, M., McQuay, H.J., Jensen, T.S. and Sindrup, S.H. (2005) Algorithm for Neuropathic Pain Treatment: An Evidence Based Proposal. Pain, 118, 289-305. http://dx.doi.org/10.1016/j.pain.2005.08.013

[7] Kikuchi, S. (2008) New Concept for Backache: Biopsychosocial Pain Syndrome. European Spine Journal, 17, 421427. http://dx.doi.org/10.1007/s00586-008-0747-1

[8] Arimura, T., Komiyama, H. and Hosoi, M. (1997) Pain Disability Assessment Scale: A Simplified Scale for Clinical Use. Japanese Journal of Behavior Therapy, 23, 7-15. (In Japanease)

[9] Yamashiro, K., Arimura, T., Iwaki, R., Jensen, M.P., Kubo, C. and Hosoi, M. (2011) A Multidimensional Measure of Pain Interference: Reliability and Validity of the Pain Disability Assessment Scale. Clinical Journal of Pain, 27, 338-343. http://dx.doi.org/10.1097/AJP.0b013e318204858a

[10] Kitamura, T. (1993) The Hospital Anxiety and Depression Scale. Archives of Psychiatric Diagnostics and Clinical Evaluation, 4, 371-372. (In Japanese)

[11] Zigmond, A.S. and Snaith, R.P. (1983) The Hospital Anxiety And Depression Scale. Acta Psychiatrica Scandinavica, 67, 361-370. http://dx.doi.org/10.1111/j.1600-0447.1983.tb09716.x

[12] Matsuoka, H. and Sakano, Y. (2007) Assessment of Cognitive Aspect of Pain: Development, Reliability, and Validation of Japanese Version of Pain Catastrophizing Scale. Japanese Journal of Psychosomatic Medicine, 47, 95-102. (In 
Japanese)

[13] Sullivan, M.J.L. and Bishop, S.R. (1995) The Pain Catastrophizing Scale. Development and Validation. Psychological Assessment, 7, 524-532. http://dx.doi.org/10.1037/1040-3590.7.4.524

[14] Japanese EuroQol Translation Team (1997) The Development of the Japanese EuroQol Instrument. Iryo to Shakai, 8, 109-123. (In Japanese)

[15] Dolan, P. (1997) Modeling Valuations for EuroQol Health States. Medical Care, 35, 1095-1108. http://dx.doi.org/10.1097/00005650-199711000-00002

[16] Jamison, R.N. and Edwards, R.R. (2012) Integrating Pain Management in Clinical Practice. Journal of Clinical Psychology in Medical Settings, 19, 49-64. http://dx.doi.org/10.1007/s10880-012-9295-2

[17] Fishman, S.M., Ballantyne, J.C. and Rathmell, J.P. (2009) Bonica’s Management of Pain. 4th Edition, Lippincott Williams \& Wilkins, Philadelphia.

[18] Kamper, S.J., Apeldoorn, A.T., Chiarotto, A., Smeets, R.J., Ostelo, R.W., Guzman, J. and van Tulder, M.W. (2015) Multidisciplinary Biopsychosocial Rehabilitation for Chronic Low Back Pain: Cochrane Systematic Review and MetaAnalysis. British Medical Journal, 350, h444.

[19] Inoue, M., Inoue, S., Ikemoto, T., Arai, Y.C., Nakata, M., Miyazaki, A., Nishihara, M., Kawai, T., Hatakeyama, N., Yamaguchi, S., Shimo, K., Miyagawa, H., Hasegawa, T., Sakurai, H., Hasegawa, Y., Omichi, Y. and Ushida, T. (2014) The Efficacy of a Multidisciplinary Group Program for Patients with Refractory Chronic Pain. Pain Research \& Management, 19, 302-308.

[20] Lane, R.D. (2014) Is It Possible to Bridge the Biopsychosocial and Biomedical Models? BioPsychoSocial Medicine, 8, 3. http://dx.doi.org/10.1186/1751-0759-8-3

\section{Abbreviations}

- FAI: Femoacetabular Impingement

- MRI: Magnetic Resonance Imaging

- NRS: Numerical Rating Scale

- PDAS: Pain Disability Assessment Scale

- HADS: Hospital Anxiety and Depression Scale

- PCS: Pain Catastrophizing Scale

- EQ-5D: EuroQol 5 Dimension

- CBT: Cognitive Behavioral Therapy 